\title{
Emergency medical services
}

\section{INTRODUCTION}

\section{Emergency medical services: groaning while growing}

\author{
John Ross, MD
}

$\mathrm{E}$

mergency medical services (EMS) have matured and grown in sophistication over the last 20 years. "Scoop and run" ground ambulance services, once a sideline business for local funeral homes, have gradually been replaced by highly organized tiered-response systems using fire departments, police and ambulance services. Now, 8- to 24-month college courses are required, and paramedics range from basic ambulance attendants to knowledgeable, multi-skilled emergency medicine providers.

Computer-aided central dispatch and medical priority dispatch have further refined the use of human and hardware resources and have helped establish standards for initial emergency care. EMS is no longer delivered from the back of a converted Cadillac, but there are foot and bicycle responders for crowds and disasters, and rotary- or fixedwing programs for scene and interfacility transports. All these developments are intended to improve public safety, provide early out-of-hospital care, and reduce morbidity and mortality. All have evolved as a result of, or in spite of, financial restrictions and restructuring.

A frequent complaint is that EMS hardware, protocols,

Dalhousie University, Halifax, NS treatments, and spiraling costs have developed in the absence of meaningful evidence to support them. Randomized controlled trials are needed, although these are difficult in the chaotic EMS environment. It is heartening that EMS directors are now encouraging utilization studies and clinical research at a local level, and that larger studies such as OPALS (Ontario Prehospital Advanced Life Support) are generating important data to support or discredit what were, until recently, intuitive precepts.

Another concern is that EMS providers are metastasizing into the traditional nursing and physician strongholds as "expanded-scope providers" or "physician assistants." Is this justified? Is it "cost effective"? Are there turf infringements?

Every Canadian jurisdiction has different geography, demography and resources; therefore EMS has evolved differently from place to place. EMS has demonstrated plasticity - the ability to change with the times and respond to both real and perceived needs. The following two authors, Dr. Karen Wanger from British Columbia and Dr. David Petrie from Nova Scotia, share their thoughts on the current and future status of EMS.

Correspondence to: johnross@is.dal.ca 